\title{
Engineering bifunctionality
}

\section{By Kai-Jye Lou, Staff Writer}

McGill University researchers report they have used a nonlinkerbased approach to build triciferol, a hybrid trichostatin A and vitamin $\mathrm{D}_{3}$ antiproliferative compound that targets histone deacetylase and the nuclear vitamin $\mathrm{D}$ receptor simultaneously and also shows in vitro efficacies comparable or superior to either strategy alone.

Although the bifunctional molecule circumvents efficacy and dosing issues typically encountered with linked compounds or combination therapies, companies contacted by SciBX said they would now like to see in vivo confirmatory data.

Activating the nuclear vitamin $\mathrm{D}$ receptor (VDR) can modulate gene transcription by regulating histone acetylation and other factors. ${ }^{1}$ In addition, analogs of the biologically active metabolite of vitamin $\mathrm{D}, 1,25$-dihydroxyvitamin $\mathrm{D}_{3}(1,25 \mathrm{D})$, which is a VDR agonist, have shown therapeutic potential in hyperproliferative disorders such as cancer and psoriasis. ${ }^{1,2}$

Meanwhile, previous reports have shown that histone deacetylase (HDAC) inhibitors can resensitize cancer cells that are resistant to the antiproliferative effects of vitamin D agonists. ${ }^{1}$

As reported in the Proceedings of the National Academy of Sciences, the researchers used existing structural and activity data from two HDAC inhibitors (trichostatin A (TSA) and Zolinza vorinostat), $1,25 \mathrm{D}$ and several $1,25 \mathrm{D}$ analogs to guide the rational design and synthesis of triciferol.

The resulting compound combined the secosteroidal backbone of $1,25 \mathrm{D}$, which is needed to activate the VDR, with the dienyl hydroxamic acid group of TSA, which inhibits HDAC. This was accomplished by replacing the side chain of $1,25 \mathrm{D}$ with the TSA moiety.

Zolinza vorinostat is marketed by Merck \& Co. Inc. to treat cutaneous $\mathrm{T}$ cell lymphoma (CTCL) and is the only approved HDAC inhibitor. The drug is also in multiple clinical trials for other cancers.

The common approach to joining molecules of different activities to generate a bifunctional agent is to use a linker molecule such as polyethylene glycol (PEG). In the case of triciferol, however, the bifunctional agent is the result of structural integration of both compounds into a new, untethered scaffold.

Avoiding the linker was important, said James Gleason, a principal investigator and a corresponding author for the PNAS manuscript and an associate professor of chemistry at McGill. "You cannot use a linker for VDR activation because the nuclear receptor needs to completely envelop its agonist," he said. "If a linker were used, there is a good chance that the resulting molecule will be too large and thus lose its ability to activate the nuclear receptor."

A 2007 review paper in Drug Discovery Today cited multiple studies that showed how increased molecular weight and complexity negatively affect ligand efficacy and oral bioavailability. ${ }^{3}$

\section{The power of two}

A reporter-gene assay showed that triciferol had VDR agonist activity that was comparable to $1,25 \mathrm{D}$. The profile of gene regulation in human squamous carcinoma cells treated with triciferol was similar to cells treated with the combination of $1,25 \mathrm{D}$ and TSA.

In four cancer cell lines including squamous carcinoma cells, triciferol produced dose-dependent reductions in cell proliferation similar to combination treatment with $1,25 \mathrm{D}$ and TSA. Fluorescence microscopy in cancer cells showed that treatment with triciferol induced multinucleation and the formation of intercellular tubulin bridges, which are the hallmarks of disrupted cell division caused by treatment with the combination of $1,25 \mathrm{D}$ and TSA.

Individually, neither TSA nor $1,25 \mathrm{D}$ induced such morphological changes.

A separate microscopy study in human MCF-7 breast cancer cells suggested that triciferol lowers cell proliferation by increasing the formation of autophagosomes and inducing autophagy. ${ }^{4}$

John White, professor of physiology and medicine at McGill and a principal investigator on the PNAS paper, added that the therapeutic windows for triciferol's VDR agonist and HDAC inhibitor activities have a high degree of overlap. This could be important, as adjusting the dose of a bifunctional compound will affect the activity against both targets. Indeed, a prime challenge of discovering new multitargeted molecules is achieving the right balance of activity between targets and maintaining favorable pharmacokinetics and oral bioavailability. ${ }^{4}$

"With a combination of two selective agents, it may be easier to optimize the treatment regimen based on the needs of an individual patient since it is possible to titrate each activity up or down," noted Richard Morphy, section head of the medicinal chemistry department at Schering-Plough Corp. "With a drug that hits multiple targets, you will need to decide at the discovery phase how much activity you want on each target. The ratio is then fixed."

He added, "Multitarget projects often require more resources, which means you should critically assess the attractiveness from a disease-based perspective and the feasibility from a medicinal chemistry perspective at the outset."

Alan Colowick, CEO of HDAC company Gloucester Pharmaceuticals Inc., suggested that the bifunctional nature of a compound can potentially limit the types of cancers that it can be used to treat. He 


\section{DRUG PLATFORMS}

told SciBX that the activity of a bifunctional molecule against one of its targets in certain forms of cancer may not be desirable for treating other forms of cancer.

Gleason countered that "unlike in combination therapies, with hybrid molecules like triciferol one only needs to be concerned with the delivery of a single compound into the cell." Doing so can potentially reduce the resources spent on making structural modifications needed to get multiple compounds into the cell.

Both White and Gleason also said that a single-molecule compound like triciferol can simplify pharmacokinetic profiles, lower the likelihood of undesirable drug interactions and reduce dosing complexity.

Robert Hanson, a Matthews Distinguished University Professor of chemistry and chemical biology at Northeastern University, agreed with White and Gleason. He said a bifunctional, single-molecule compound will have unitary pharmacokinetics and is unlikely to interfere with its own activity.

"With compound drugs," he told SciBX, "it is more difficult to maintain fixed ratios of activity and unwanted drug-drug interactions are more likely."

\section{Bifunctional future}

White said his research group is now working with multiple bifunctional VDR agonists that show a range of inhibitory activity against various HDACs. "We have already synthesized multiple bifunctional molecules wherein $100 \%$ of the molecule is a VDR agonist and up to $100 \%$ of the molecule is an HDAC inhibitor," he said.

Moreover, said White, the molecules have varying specificity for different HDACs, which will be important for dose selection and the ability of the compounds to treat other diseases.

The group will shortly begin evaluating triciferol and several of the other bifunctional molecules in tumor xenograft models of cancer.

White noted the applicability of triciferol beyond cancer to diseases like psoriasis and certain forms of arthritis.

"To determine the therapeutic potential of triciferol, it will be important to evaluate its activity in in vivo models," said Victoria Richon, senior director of cancer research at Merck. "This and additional studies are required to determine the benefit of this multiple-ligand approach compared to each individual agent and the combination of the two agents."
Gloucester's Colowick also told SciBX that "the theoretical advantages and theoretical disadvantages of bifunctional molecules like triciferol cannot be accurately assessed without in vivo data."

Last month, the company reported positive Phase IIb data for its HDAC inhibitor Romidepsin in CTCL. The company expects to submit an NDA for the drug later this year. Romidepsin is also in a pivotal Phase II trial to treat peripheral $\mathrm{T}$ cell lymphoma (PTCL).

Morphy said he wanted to see tests for off-target effects and estimates for the cost of synthesis. Like Richon and Colowick, he thinks that to increase the appeal of triciferol to biotech companies, it would be necessary to evaluate the compound in vivo and show favorable pharmacokinetic profiles and oral bioavailability data.

"Feedback from the clinic is invaluable for determining the optimal level of activity for each target to maximize efficacy and safety," Morphy said. "This could be derived from proof-of-concept studies using variable dose combinations of single-target drugs or from firstin-class multitarget drugs, if available."

Similarly, Hanson wanted to see additional data on how triciferol's lipophilicity and polarity will affect its absorption and bioavailability in vivo. He also recommended the researchers attempt to reduce the number of synthesis steps to reduce the compounds' cost.

Patent applications have been filed for triciferol and the related bifunctional molecules. The compounds are available for licensing through McGill.

\section{REFERENCES}

1. Lin, R. \& White, J.H. BioEssays 26, 21-28 (2004)

2. Masuda, S. \& Jones, G. Mol. Cancer. Ther. 5, 797-808 (2006)

3. Morphy, R. \& Rankovic, Z. Drug Discov. Today 12, 156-160 (2007)

4. Tavera-Mendoza, L.E. et al. Proc. Nat. Acad. Sci. USA; published online June 09, 2008; doi:10.1073/pnas.0709279105

Contact: John H. White, McGill University, Montreal, Quebec, Canada e-mail: john.white@mcgill.ca

Contact: James L. Gleason, same affiliation as above e-mail: jim.gleason@mcgill.ca

\section{COMPANIES AND INSTITUTIONS MENTIONED}

Gloucester Pharmaceuticals Inc., Cambridge, Mass.

McGill University, Montreal, Quebec, Canada

Merck \& Co. Inc. (NYSE:MRK), Whitehouse Station, N.J.

Northeastern University, Boston, Mass.

Schering-Plough Corp. (NYSE:SGP), Kenilworth, N.J. 